\title{
Long spin-flip time and large Zeeman splitting of holes in type-II ZnTe/ZnSe submonolayer quantum dots
}

\author{
H. Ji, S. Dhomkar, R. Wu, J. Ludwig, Z. Lu, D. Smirnov, M. C. Tamargo, G. W. Bryant, and I. L. Kuskovsky
}

Citation: Journal of Applied Physics 124, 144306 (2018); doi: 10.1063/1.5041478

View online: https://doi.org/10.1063/1.5041478

View Table of Contents: http://aip.scitation.org/toc/jap/124/14

Published by the American Institute of Physics

\section{Articles you may be interested in}

Analytical modeling of temperature and power dependent photoluminescence (PL) spectra of InAs/GaAs quantum dots

Journal of Applied Physics 124, 145701 (2018); 10.1063/1.5047026

A thermoacoustic imaging system with variable curvature and multi-dimensional detection adapted to breast tumor screening

Journal of Applied Physics 124, 144902 (2018); 10.1063/1.5042121

Photothermal effect in solid-state MWCNT: Possible signatures of thermal anisotropy

Journal of Applied Physics 124, 145104 (2018); 10.1063/1.5030461

Fundamental exciton transitions in $\mathrm{SiO}_{2} / \mathrm{Si} / \mathrm{SiO}_{2}$ cylindrical core/shell quantum dot

Journal of Applied Physics 124, 144303 (2018); 10.1063/1.5047057

Energy filtering in silicon nanowires and nanosheets using a geometric superlattice and its use for steep-slope transistors

Journal of Applied Physics 124, 144304 (2018); 10.1063/1.5043543

Transport properties and thermoelectric effects in gated silicene superlattices

Journal of Applied Physics 124, 144305 (2018); 10.1063/1.5045479

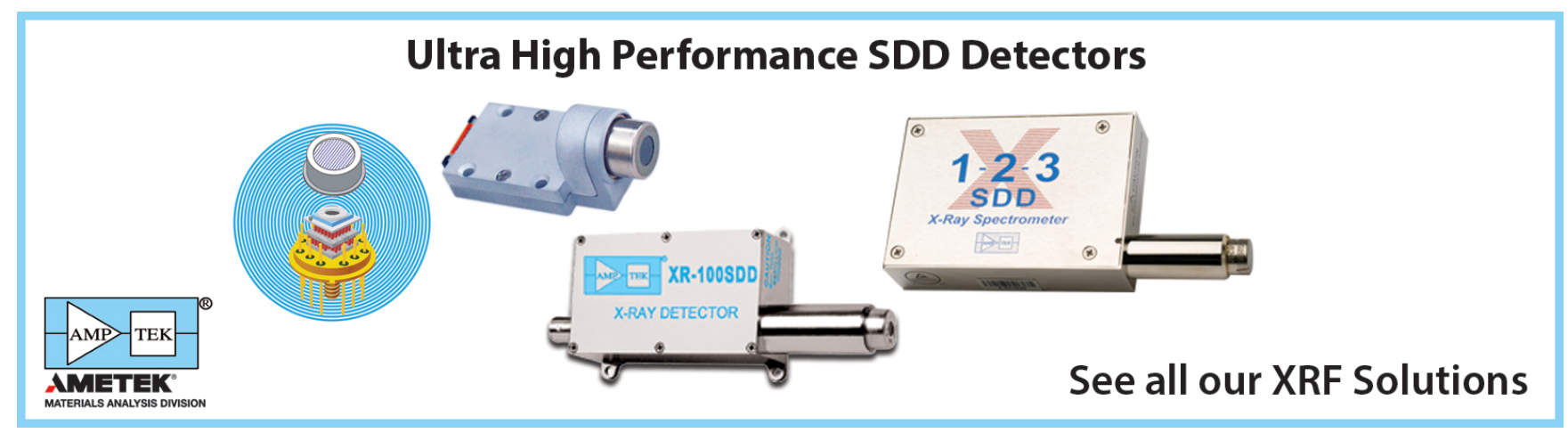




\title{
Long spin-flip time and large Zeeman splitting of holes in type-II ZnTe/ZnSe submonolayer quantum dots
}

\author{
H. Ji, ${ }^{1,2, a)}$ S. Dhomkar, ${ }^{1,2}$ R. Wu, ${ }^{1,2}$ J. Ludwig, ${ }^{3,4}$ Z. Lu, ${ }^{3,4}$ D. Smirnov, ${ }^{3}$ M. C. Tamargo, ${ }^{2,5}$ \\ G. W. Bryant, ${ }^{6}$ and I. L. Kuskovsky ${ }^{1,2, b)}$ \\ ${ }^{1}$ Department of Physics, Queens College of CUNY, Queens, New York 11367, USA \\ ${ }^{2}$ The Graduate Center of CUNY, New York, New York 10016, USA \\ ${ }^{3}$ National High Magnetic Field Laboratory, Tallahassee, Florida 32310, USA \\ ${ }^{4}$ Department of Physics, Florida State University, Tallahassee, Florida 32306, USA \\ ${ }^{5}$ Department of Chemistry, City College of CUNY, New York, New York 10031, USA \\ ${ }^{6}$ Quantum Measurement Division and Joint Quantum Institute, National Institute of Standards and Technology, \\ Gaithersburg, Maryland 20899-8423, USA
}

(Received 25 May 2018; accepted 22 September 2018; published online 11 October 2018)

\begin{abstract}
The Zeeman splitting and degree of circular polarization (DCP) of photoluminescence (PL) from type-II submonolayer ZnTe/ZnSe quantum dots (QDs) have been investigated in magnetic fields up to $18 \mathrm{~T}$. To explain the observed relative intensities and energy positions of the $\sigma^{+}$and the $\sigma^{-} \mathrm{PL}$, a non-Boltzmann distribution for holes with ultra-long spin-flip time, confined to submonolayer QDs, is proposed. The $g$-factor of electrons, located in the $\mathrm{ZnSe}$ barriers, was obtained from fitting the temperature dependence of the DCP, and its value is in excellent agreement with that of bulk $\mathrm{ZnSe}$. The $g$-factor of type-II excitons was extracted by analyzing the Zeeman splitting, from which the $g$-factor of holes confined within submonolayer ZnTe QDs was found to be $\sim 2.65 \pm 0.40$. This value is considerably larger than that in bulk ZnTe. Tight-binding calculations using an $s p^{3} s^{*}$ model were employed to understand the origin of such an increase. The results of the simulation match the experiment and show that the enhancement of the hole $g$-factor is mostly caused by a reduced orbital contribution to Zeeman splitting arising from the submonolayer thickness of these QDs. Published by AIP Publishing. https://doi.org/10.1063/1.5041478
\end{abstract}

\section{INTRODUCTION}

In recent years, there has been intense interest in manipulating electron and hole spin states in semiconductor quantum dots (QDs) for applications in spintronics and quantum information processing. ${ }^{1-5}$ For these applications, the confinement of the wavefunction of the charge carriers in QDs plays a key role in the enhancement of Zeeman splitting $^{6,7}$ and the prolongation of spin-flip time. ${ }^{8,9}$ These effects have been reported for electrons in several material systems. ${ }^{10-15}$ For holes confined in QDs, theoretical studies have predicted that its spin-flip time can be longer than for confined electrons. ${ }^{16-19}$ Along with the enhancement of the Zeeman splitting due to the quantum confinement, holes in QDs have attracted increased attention as carriers for spinbased applications. However, experimental results of $g$-factors or spin-flip time of holes in II-VI QD systems are rarely reported.

Herein we report the discovery of a very long spin-flip time and large enhancement of Zeeman splitting for the holes confined in type-II ZnTe/ZnSe submonolayer QDs, supporting the use of holes in these QDs for spintronic and quantum information applications. The $g$-factors of excitons, electrons, and holes in type-II submonolayer $\mathrm{ZnTe} / \mathrm{ZnSe}$ QDs were obtained by studying circularly polarized magnetophotoluminescence (magneto-PL). The $g$-factor of the

\footnotetext{
a)jihaojie.jhj@gmail.com

b)Igor.Kuskovsky@qc.cuny.edu
}

QD-bound type-II excitons (with holes confined in ZnTe QDs and electrons located in ZnSe barriers) was extracted from the Zeeman splitting. The observed energy and intensity relations between the $\sigma^{+}$and the $\sigma^{-}$polarized emissions are explained by a non-Boltzmann distribution model, with a positive $g$-factor for holes and a hole spin-flip time longer than the recombination lifetime of excitons, which for ZnTe/ $\mathrm{ZnSe}$ QDs is more than $100 \mathrm{~ns}$. Within this model, the $g$-factor of electrons was estimated from the temperature dependence of the degree of circular polarization (DCP) and was shown to agree well with values available in the literature. The $g$-factor of holes confined within ZnTe QDs was then calculated from the $g$-factors of excitons and electrons, and was found to be positive and one order of magnitude larger than the values reported for bulk ZnTe. Tight-binding calculations based on an $s p^{3} s^{*}$ model were employed to theoretically determine the $g$-factor of the holes confined in $\mathrm{ZnTe} / \mathrm{ZnSe}$ QDs. The results of the simulation are in excellent agreement with the experimentally determined values. The calculations showed that the enhancement of the $g$-factor of holes is mostly caused by a reduced orbital contribution to Zeeman splitting due to the submonolayer nature of the QDs.

\section{EXPERIMENTAL RESULTS AND DISCUSSION}

The type-II ZnTe/ZnSe stacked submonolayer QDs investigated here are grown using a combination of migration-enhanced epitaxy and molecular beam epitaxy (see Refs. 20 and 21 and the references therein). Circularly 


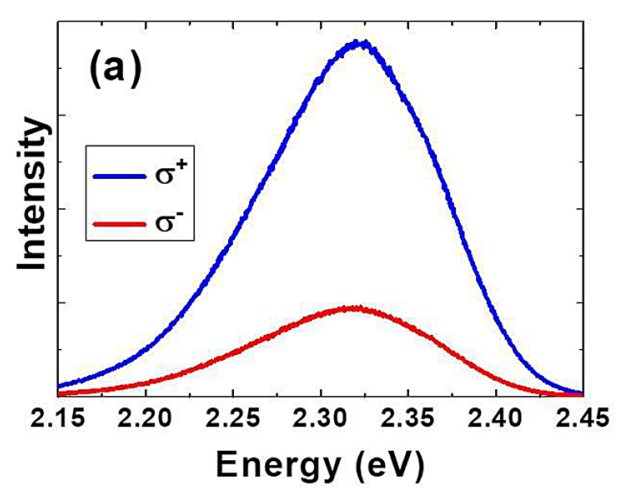

(c)

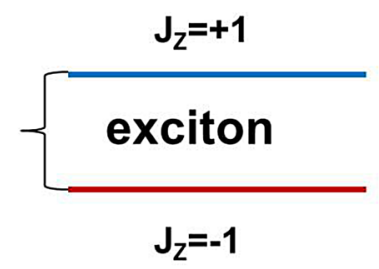

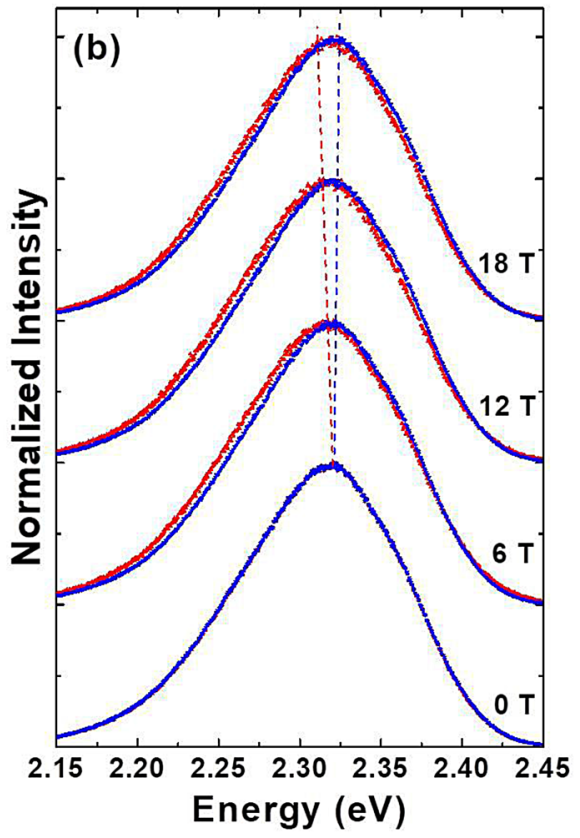

FIG. 1. (a) PL spectra at $0.36 \mathrm{~K}$ of $\sigma^{+}$ and $\sigma^{-}$polarized emission under a magnetic field of $18 \mathrm{~T}$. (b) Normalized PL spectra under different magnetic field at $0.36 \mathrm{~K} . \sigma^{+}$is shown in blue while $\sigma^{-}$is shown in red. The dashed lines trace the peaks of the spectra at increasing magnetic fields. (c) Zeeman splitting of the two exciton spin states. polarized magneto-PL measurements were performed at the National High Magnetic Field Laboratory at temperatures between $0.36 \mathrm{~K}$ and $30 \mathrm{~K}$ with magnetic fields up to $18 \mathrm{~T}$ applied in the Faraday geometry (see Ref. 22 for more details). The $405 \mathrm{~nm}$ continuous wave (cw) light excitation from a temperature controlled laser diode was injected into a $365 \mu \mathrm{m}$ fiber and delivered to the sample in a ${ }^{3} \mathrm{He}$ cryostat. The PL was analyzed by a circular polarizer consisting of an achromatic quarter wave plate and a linear polarizer. The $\sigma^{+}$ and $\sigma^{-}$circularly polarized PL components were selected by reversing the polarity of the magnetic field. The collected PL was injected into a $550 \mu \mathrm{m}$ fiber and delivered to a single grating spectrometer equipped with a thermoelectrically cooled CCD detector.

Figure 1(a) shows the PL spectra at $0.36 \mathrm{~K}$ of $\sigma^{+}$and $\sigma^{-}$ polarized emission at a magnetic field of $18 \mathrm{~T}$. An important observation is that the intensity of $\sigma^{+}$polarized emission is higher than that of $\sigma^{-}$polarized emission. To better observe the energy difference between $\sigma^{+}$and $\sigma^{-}$polarized emissions, we plot the normalized $\sigma^{+}$and $\sigma^{-}$PL for selected values of magnetic field at $\mathrm{T}=0.36 \mathrm{~K}$ in Fig. 1(b). As expected, at $\mathrm{B}=0 \mathrm{~T}$, the differently polarized bands coincide. With increasing magnetic field, the spectrum of the $\sigma^{+}$polarized emission shifts to higher energies while that of the $\sigma^{-}$ polarized emission shifts to lower energies. We note that while the observed shift is much smaller than the width of the PL, the shift is clear as it pertains to a whole band and not only to its maximum. Therefore, we observed the relatively rare case where $\sigma^{+}$polarized emission with higher energy shows higher emission intensity, which we will discuss below.

We start from the energy relation between $\sigma^{+}$and $\sigma^{-}$ polarized emissions. A schematic diagram of the Zeeman splitting of the excitons' spin states which coincide with the observed relative energies of $\sigma^{+}$and $\sigma^{-}$polarized emission is shown in Fig. 1(c). For the type-II excitons, the $g$-factor of electrons located in the ZnSe barrier is positive. ${ }^{23}$ The holes are confined in the ZnTe QDs. The ground state of holes confined in QDs is the heavy hole like state, ${ }^{24,25}$ which has spin $J_{z}= \pm \frac{3}{2}$, with at least $200 \mathrm{meV}$ energy splitting between heavy and light hole energy levels. ${ }^{24}$ Based on optical selection rules, as shown in Fig. 2, the bright exciton with $J_{Z}=-1$ consists of a spin-up electron and a spin-down hole whose recombination will emit a $\sigma^{-}$photon, while that with $J_{Z}=+1$ consists of a spin-down electron and a spin-up hole whose recombination will emit a $\sigma^{+}$photon. Therefore, since the spin-up $\left(J_{Z}=+1\right)$ excitons have higher energy than the
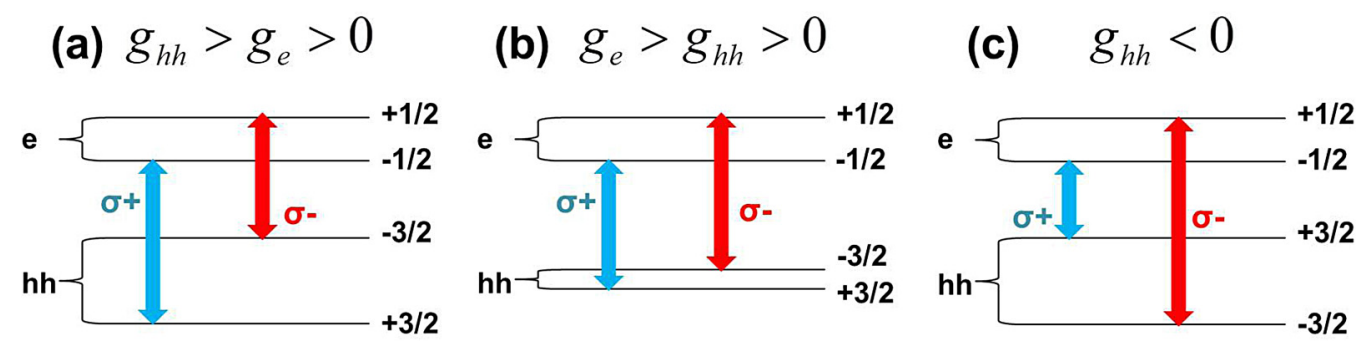

FIG. 2. Zeeman splitting of the ground states of electron and heavy hole for the case of (a) $g_{h h}>g_{e}>0$, (b) $g_{e}>g_{h h}>0$, and (c) $g_{h h}<0$. The doublearrows indicate the spin states associated with $\sigma^{+}$and $\sigma^{-}$polarized emission, corresponding to the $J_{Z}=+1$ and $J_{Z}=-1$ excitons, respectively. Here, the valence band states are in hole representation. 


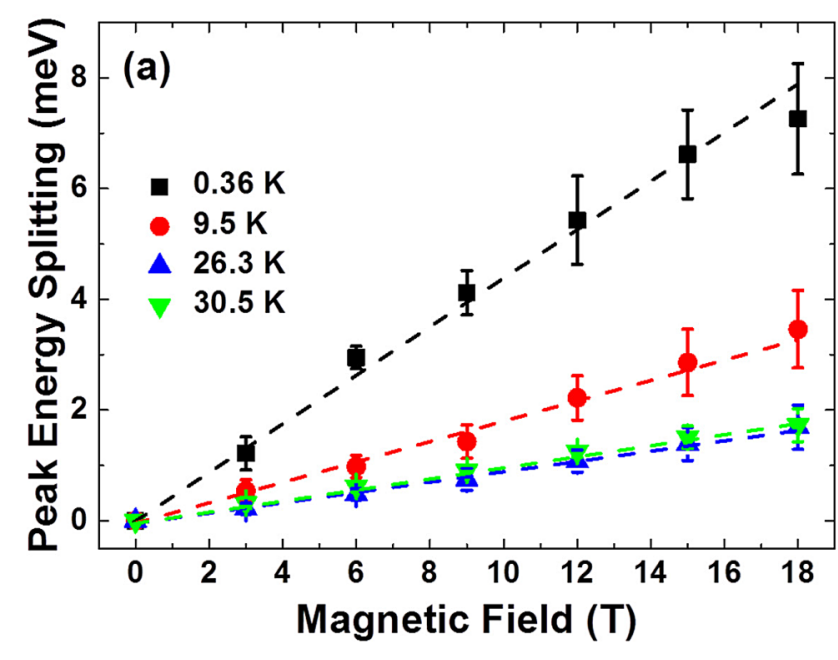

spin-down $\left(J_{Z}=-1\right)$ excitons, the $g$-factor of the excitons is positive, as the energy splitting between the $\sigma^{+}$and the $\sigma^{-}$ polarized spectra, given by ${ }^{26}$

$$
E_{\sigma^{+}}-E_{\sigma^{-}}=g_{h h} \mu_{B} B-g_{e} \mu_{B} B=g_{X} \mu_{B} B,
$$

is positive in our case (here $g_{h h}, g_{e}$, and $g_{X}$ are the $g$-factors of heavy holes, electrons, and the type-II excitons; $\mu_{B}$ is the Bohr magneton and $B$ is the magnetic field applied in the Faraday geometry).

Since the $g$-factor of electrons, located in $\mathrm{ZnSe}$ barriers, is positive $^{23}$ (see also discussion below), the spin-up electrons have higher energy than the spin-down electrons. We therefore show in Fig. 2 all possible situations of sign and relative magnitude of the heavy holes $g$-factor. According to both Eq. (1) and Fig. 2, the heavy holes $g$-factor in ZnTe QDs ought to be positive and larger than the $g$-factor of electrons in $\mathrm{ZnSe}$ to ensure that excitons with $J_{Z}=+1$ have higher energy than those with $J_{Z}=-1$.

To find the value of the $g_{X}$ in Eq. (1), we plot the average peak energy splitting between $\sigma^{+}$and $\sigma^{-}$polarized spectra, extracted from a Gaussian fitting of each polarized PL band, as a function of magnetic field at different temperatures in Fig. 3(a). The errors are determined by combining the uncertainty of fitting and the difference between the results of several sets of measurements, and they are one order of magnitude smaller than the values of energy splitting. The errors are mostly related to the inhomogeneity of the QD sample, since the excitations were on different regions of the sample, so that the polarized PL were collected from different groups of QD stacks for different sets of measurements. This agrees with the fact that the $g$-factors are dependent on the size of QDs, which will be shown later by the calculations. Therefore, the average peak energy splitting extracted from a Gaussian fitting corresponds to the average $g$-factors of different sets of QDs. However, it is surprising that the splitting at lower temperatures is much larger than the splitting at higher temperatures since the $g$-factors should not have strong temperature dependence. ${ }^{27}$

We attribute this observation to the multiple-band nature of the PL spectra in our samples as discussed previously in Refs. 20 and 28. To confirm this, we fitted each polarized PL

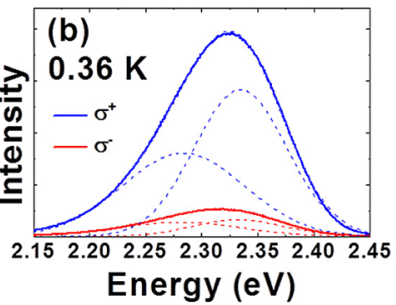

FIG. 3. (a) Average peak energy splitting with error bars between $\sigma^{+}$and $\sigma^{-}$ polarized spectra as a function of magnetic field of the superconducting magnet at different temperatures. Dashed lines are linear fits. (b) and (c) PL spectra of $\sigma^{+}$and $\sigma^{-}$polarized emission under magnetic field $18 \mathrm{~T}$ at 0.36 and $30.5 \mathrm{~K}$, respectively. The dashed lines are fittings with two Gaussian bands.

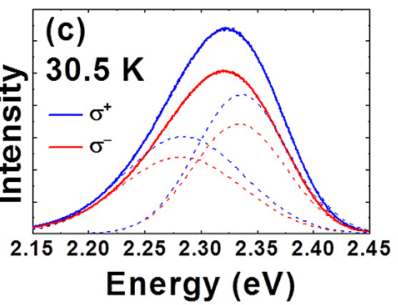

spectrum with two internal Gaussian bands as shown in Fig. 3(b) as an example for $18 \mathrm{~T}$ at $0.36 \mathrm{~K}$. According to our previous study of the multi-band nature of the submonolayer ZnTe/ZnSe QDs, ${ }^{20,21,25,28}$ the more Gaussian bands we used to fit the PL spectra, the better match of the fitted curves and the observed PL line shape. However, the more Gaussian bands used in the fitting, the more sensitive the fitting parameters to the initial conditions, which makes the fitting less reliable. Therefore, on balance we always use two Gaussian bands to represent two different size-groups (larger ones and smaller ones) of QDs. The peak energy difference between the two internal Gaussian bands for each polarization is about $50 \mathrm{meV}$, comparable to the expected energy difference between a lower and upper band for different QD sizes. The peak energy difference between the corresponding internal Gaussian bands for $\sigma^{+}$and $\sigma^{-}$emission is $\sim 2 \mathrm{meV}$, the same as the peak energy difference between the full polarized spectra at $30.5 \mathrm{~K}$ as shown in Fig. 3(c). However, since the $D C P$, defined as $\frac{I_{\sigma^{+}}-I_{\sigma^{-}}}{I_{\sigma^{+}}+I_{\sigma^{-}}}$, of the internal Gaussian band with a higher energy is larger than that of the internal Gaussian band with a lower energy at low temperatures, the overall $\sigma^{+}$spectrum is effectively shifted to higher energies at low temperatures. Therefore, the apparent energy splitting of the polarized spectra at $0.36 \mathrm{~K}$ is effectively enhanced. The fact that the PL band with a lower energy is less circularly polarized agrees with our previous work on the optical anisotropy of the ZnTe QDs. ${ }^{28}$ Indeed, the internal Gaussian PL bands with lower energy correspond to larger QDs, which are characterized by larger structure anisotropy, resulting in a stronger mixing of the circularly polarized exciton states and lowering the DCP. ${ }^{28}$ With increasing temperature, the DCP of all bands decreases, so that the related contributions to the peak energy splitting are reduced and the energy differences eventually saturate. The saturation is indeed observed above $26 \mathrm{~K}$, so the magnetic field dependences of peak energy splitting coincide, as seen in Fig. 3(a). Therefore, the peak energy splitting via one Gaussian fitting at relatively high temperatures is assumed to relate purely to the average Zeeman splitting of the spin states. We use Eq. (1) to fit the magnetic field dependence of peak energy splitting between $\sigma^{+}$and $\sigma^{-}$polarized spectra at 26.3 and 
$30.5 \mathrm{~K}$. The fitting gives the average $g_{X}=1.65 \pm 0.3$. We emphasize here that this exciton $g$-factor and the resulting hole $g$-factor are in good agreement with the simulations, as shown later. Therefore, it supports the model that the large Zeeman splitting at low temperatures is due to the contribution of multiple bands. Otherwise, the experimentally fitted $g$-factor would be much (3-4 times) larger than the calculated results for QDs with any possible parameters.

Next we consider the origin of the difference in the intensities of $\sigma^{+}$and $\sigma^{-}$polarized emissions. Experimentally, $\sigma^{+}$polarized emission has a higher photon energy, as well as higher PL intensity. This can arise from the differences in either their recombination oscillator strengths or exciton populations of $J_{Z}=+1$ and $J_{Z}=-1$ states, since $I_{+/-} \propto P_{+/-} \times O_{+/-}$, where $I_{+/-}, P_{+/-}$, and $O_{+/-}$are the PL intensity, population, and oscillator strength of the exciton $J_{Z}=+1$ and $J_{Z}=-1$ states, respectively. ${ }^{26}$ The following discussion will show that a special population distribution of electron and hole spin states rather than the difference in recombination oscillator strengths leads to the difference in the intensities of $\sigma^{+}$and $\sigma^{-}$polarized emission.

For the conventional case of a Boltzmann distribution of the excitons in a two level system (see, e.g., Ref. 26 and references therein), the $J_{Z}=-1$ state would be more populated than the $J_{Z}=+1$ state. Therefore, the emission intensity of $\sigma^{-}$polarized emission would be higher (negative DCP), which contradicts the experimental observations [Fig. 1(a)] unless the recombination oscillator strength of the $J_{Z}=+1$ state is larger than that of the $J_{Z}=-1$ state. Even if the recombination oscillator strength of the $J_{Z}=+1$ state is larger than that of the $J_{Z}=-1$ state, there is a contradiction with experiment if the system follows a Boltzmann distribution. For increasing temperature, the population of the $J_{Z}=+1$ state will increase, resulting in a decreasing difference in the population of the two spin states for the system following a Boltzmann distribution. Because the oscillator strengths are temperature independent, the PL intensity of the $J_{Z}=+1$ state would increase, whereas the PL intensity of the $J_{Z}=-1$ state would decrease with the increasing temperature. As a result, the experimentally positive $D C P$, defined as $\frac{I_{\sigma^{+}}-I_{\sigma^{-}}}{I_{\sigma^{+}}+I_{\sigma^{-}}}$, would increase with increasing temperature and reach a maximum determined by the oscillator strength of the $J_{Z}=+1$ state. This again contradicts the experimental observation of the temperature dependence of $D C P$ shown in Figs. 3(b), 3(c), and 4(a).

Thus, we conclude that in our system the exciton occupation for $J_{Z}=+1$ and $J_{Z}=-1$ states does not follow the two-state Boltzmann distribution and that the population of the $J_{Z}=+1$ type-II excitons is higher than that of the excitons in the $J_{Z}=-1$ state. This is only possible if distributions of either electrons or holes or both do not follow the Boltzmann distribution and a spin-flip time for at least one of the carriers is longer than the observed exciton radiative recombination time, which is $40-100 \mathrm{~ns}$ in type-II $\mathrm{ZnTe} /$ ZnSe QDs. ${ }^{20}$

For further analysis, we note that the excitation laser used in the experiment was not circularly polarized and the photon energy was above the $\mathrm{ZnSe}$ bandgap, so that the photo-generated charge carriers are initially equally distributed in their spin states. The electrons are located in the $\mathrm{ZnSe}$ barriers, so it is reasonable to assume that the electron spinflip time is the same as in bulk $\mathrm{ZnSe}$, which is quite short. ${ }^{20,29}$ Thus, the electron spin states are in thermal equilibrium, following the Boltzmann distribution, before the type-II excitons are formed. Therefore, the $-1 / 2$-spin state
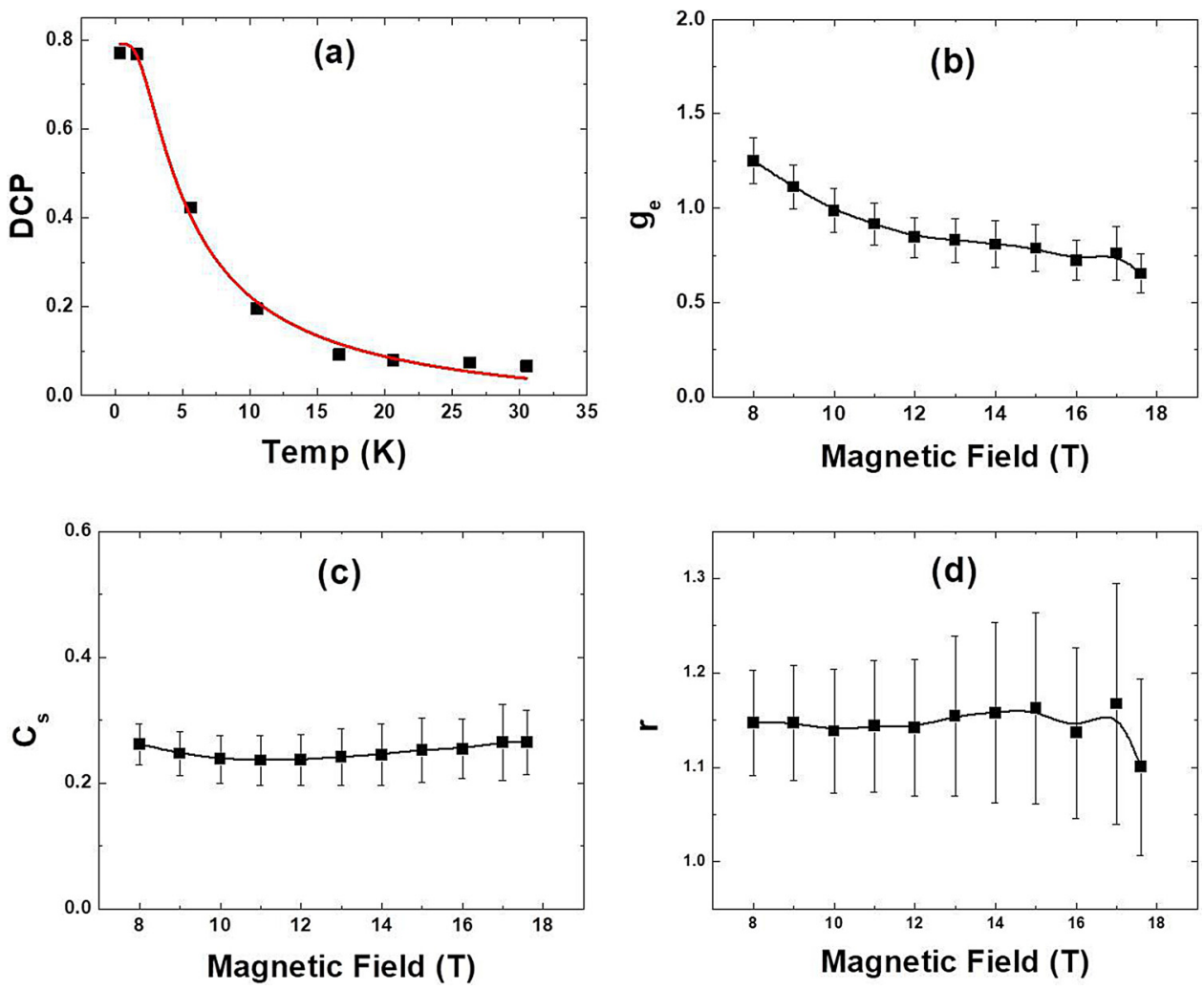

FIG. 4. (a) Temperature dependence of DCP at $10 \mathrm{~T}$. The line is fitted to Eq. (2). (b)-(d) The fitting parameters with error bars for the temperature dependence of DCP at different magnetic fields, fitted to Eq. (2). Lines are drawn to guide the eye. 

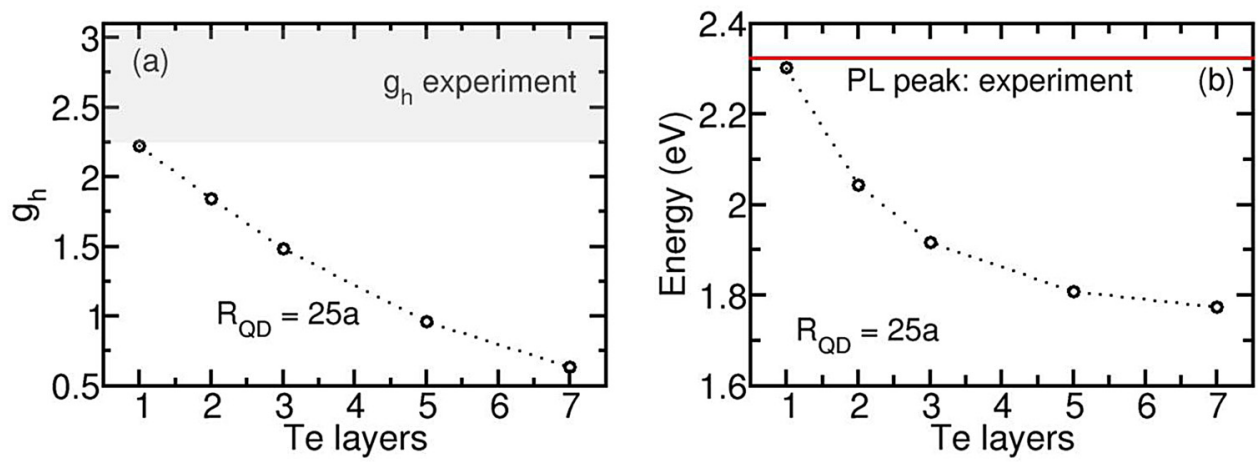

FIG. 5. (a) Dependence of the calculated hole $g$-factor on QD thickness for $R_{Q D}=25 a$. The range of possible experimental values for the hole $g$-factor is indicated by the gray region. (b) The dependence of the exciton energy on QD thickness. The red line shows the experimental PL peak energy. electrons have a higher population. As for the holes, since they are scattered into the QDs with the assistance of phonons, their spin states are assumed to be equally occupied before the formation of type-II excitons. Thus, in the beginning, there are more type-II excitons formed with $J_{Z}=+1$ than those with $J_{Z}=-1$ because there are more spin $-1 / 2$ electrons.

However, to observe the effect of such electron and hole distributions via luminescence, the distributions have to be preserved before charge carriers recombine. This is only possible if spin-flip time of holes is longer than exciton radiative recombination time (40-100 ns in type-II ZnTe/ZnSe QDs ${ }^{20}$ ). Indeed, it is predicted theoretically ${ }^{16-19}$ that the holes confined in QDs can have very long spin-flip time because of the suppression of hyperfine interaction of holes with lattice nuclei compared to that of electrons, of the weak contribution of elastic phonons to spin flip due to the discrete hole states, and the spin-orbit mixing being inhibited by the motional quantization of holes in QDs.

Finally, with increasing temperature, the Boltzmann distribution of the electron spin states approaches an equal distribution, resulting in the decrease in the difference of the population of $J_{Z}=+1$ and $J_{Z}=-1$ excitons. Therefore, the $D C P$ decreases with increasing temperature in agreement with the experimental observations.

Within the above discussed model, in which the difference of PL emission from two exciton spin states is dominated by the unequal distribution of the electron spin states, the $D C P$ can be expressed as ${ }^{30,31}$

$$
D C P=\frac{1-\exp \left[\ln (r)-\frac{\Delta E}{k T}\right]}{1+C_{s}+\exp \left[\ln (r)-\frac{\Delta E}{k T}\right]},
$$

where $\Delta E=g_{e} \mu_{B} B$ is the energy splitting of the electron spin states only; $k$ is the Boltzmann constant and $T$ is the temperature; $r$ is the ratio of recombination oscillator strengths of $\sigma^{+}$ and $\sigma^{-}$polarized emission; and $C_{S}$ is a constant that includes contributions from spin-flip time and structural anisotropy. ${ }^{30}$ The electron Zeeman-energy splitting dominates the decreasing behavior of the $D C P$ with increasing temperature at $T \sim \Delta E / k$. The ratio of recombination oscillator strengths dominates the $D C P$ at the high temperatures where the ratio of the electron (and thus exciton) populations approaches 1 . The contribution from spin-flip time and structural anisotropy dominates the saturation value of the $D C P$ in the low temperature limit. From the equation, it is clear that the stronger the structural anisotropy of the QDs, the smaller the degree of circular polarization for the emission from the QDs, supporting the previous statement that lower-energy emission bands from larger-size and more-anisotropic QDs are less circularly polarized. We use data for $10 \mathrm{~T}$ magnetic field to plot the $D C P$ as a function of temperature in Fig. 4(a), and fit it employing Eq. (2). The fitting gives $g_{e}=1.0 \pm 0.1$, $C_{s}=0.26 \pm 0.04$, and $r=1.1 \pm 0.1$, while the uncertainty is determined from the standard errors of the fitting parameters. We applied the same fitting procedure to DCPs obtained for magnetic fields higher than $8 \mathrm{~T}$, where the anisotropic splitting is overcome by the Zeeman splitting; the fitting parameters and the standard errors are consistent for different magnetic fields, as shown in Figs. 4(b)-4(d). The small ratio of the recombination oscillator strength agrees with the proposed model of long spin-flip time for holes in ZnTe submonolayer QDs, so that the large DCP is largely related to the distribution of the electron spin states. We notice that the fitted electron $g$-factor monotonically decreases with increasing magnetic field. This is explained by the contribution to
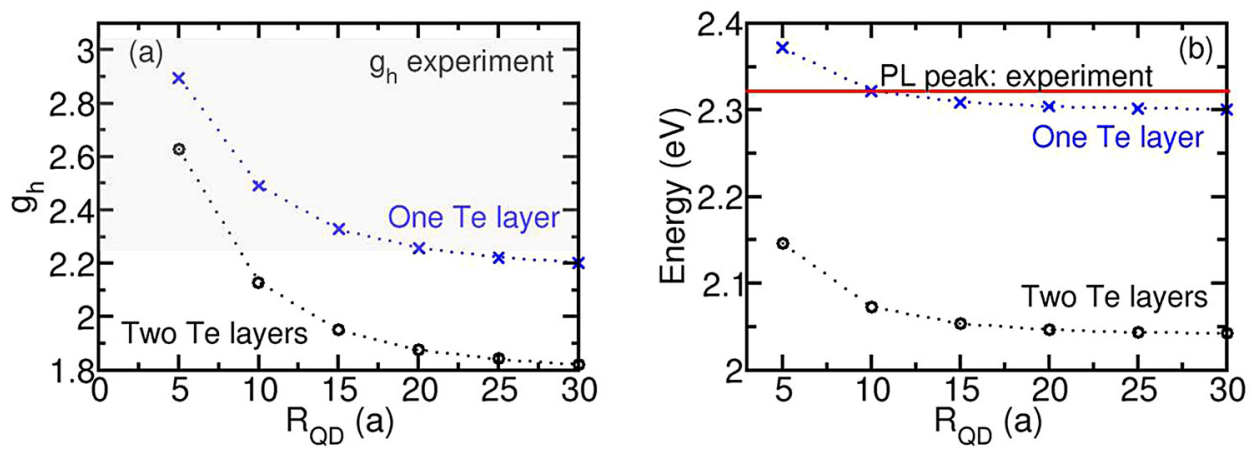

FIG. 6. (a) Influence of size fluctuations on the calculated hole $g$-factor for QDs with one or two Te layers. The range of possible experimental values for the hole $g$-factor is indicated by the shaded region. (b) The dependence of the exciton energy on QD size. The red line shows the experimental PL energy. 

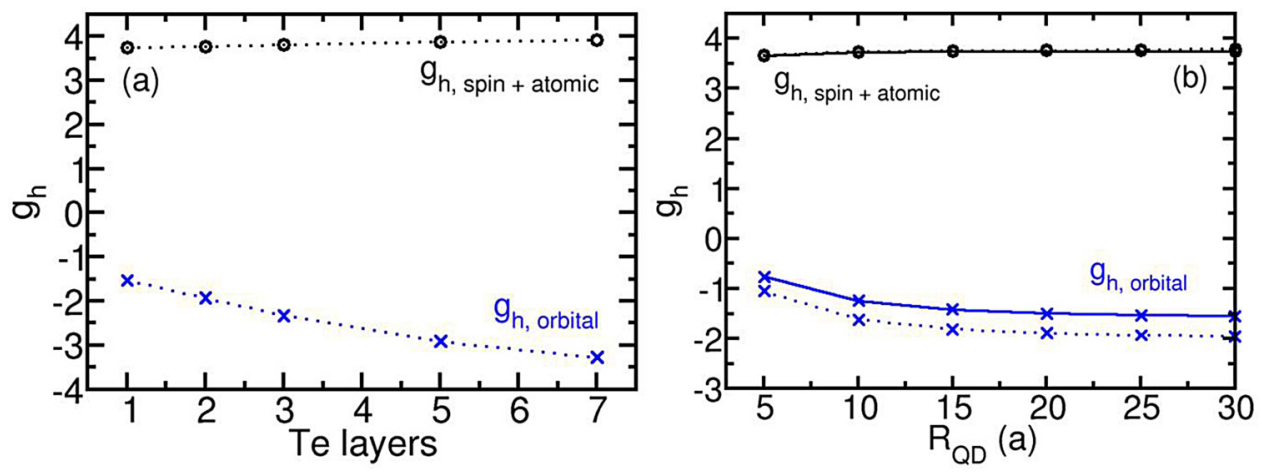

FIG. 7. (a) Contribution of hole spin, atomic orbital angular momentum, and orbital motion in the QD to the hole $g$-factor. Dependence on QD thickness for $R_{O D}=25 a$. (b) The dependence on QD radius for QDs with 1 (solid) and 2 (dotted) Te layers.

the PL from the anisotropic (nearly) dark states, with emission that is not circularly polarized. ${ }^{32}$

As defined in Eq. (1), the $g$-factor of heavy holes in QDs can be calculated as $g_{h h}=g_{X}+g_{e}=2.65 \pm 0.4$. This value is significantly larger than the hole $g$-factor in bulk ZnTe ( $g_{h h}=6 \kappa+\frac{27}{2} q=0.85$, using the formula in Ref. 26 and the Luttinger parameters from Ref. 33). To better understand the measured value of the hole $g$-factor, we performed atomistic, tight-binding calculations as described in Ref. 34. An $s p^{3} s^{*}$ model is used with strain included via the valence force field method. A constant, static magnetic field is incorporated in the tight-binding approach via a Peierls transformation, ${ }^{35}$ with interaction with atomic orbital angular momentum and spin included with an atomic Zeeman term. For ZnTe and $\mathrm{ZnSe}$, we used the tight-binding parameters from Ref. 36, adjusted to account for spin-orbit coupling that reproduces the splitting of the split off band. ${ }^{37}$ We performed calculations for disk-like QDs with thickness and diameter defined in terms of the ZnTe lattice constant, $a=6.085$. The calculations were performed for strained QDs by setting the ZnTe QD within a ZnSe matrix and relaxing the lattice of the QD and ZnSe barrier matrix into the lowest energy configuration. Results for strained QDs with 1, 2, 3, 5, and 7 layers of Te in the QD (corresponding to thicknesses of $a / 2$, $a, 3 a / 2,5 a / 2$, and $7 a / 2$, respectively) at a fixed radius of $25 a$ (the lateral size corresponds to that estimated in Ref. 25) are shown in Fig. 5. The corresponding PL emission energies are found by assuming the electron is at the $\mathrm{ZnSe}$ band edge in this type II structure, without including the exciton binding energy, which is small (on the order of $10 \mathrm{~s}$ of $\mathrm{meV}$ ) compared to the emission energy because the electron and hole are spatially separated in the type II structure. The calculated PL energies are compared with the experiment in Fig. 5(b). The energies for QDs with one Te layer are in good to excellent agreement with those we observed experimentally. For thick QDs, the predicted $g$-factor for the ground state (heavy) holes falls well below the experimental value. However, as the QD thickness is reduced, the predicted hole $g$-factor increases and approaches the experimental value.

At this QD radius, the hole $g$-factor is still underestimated, except for the thinnest QDs. However, for QDs as thin as one or two layers of Te, fluctuations in the thickness of the QD should have a substantial effect. To test the effect of these size fluctuations, we have calculated the hole $g$-factor for QDs with 1 or 2 layers of Te and different $R_{Q D}$.
As shown in Fig. 6, for $R_{Q D}$ where the predicted exciton energy matches the measured PL peak energy, the hole $g$-factor is close to the experimental result.

The $g$-factor for the ground state (heavy) holes is a sensitive function of both the QD thickness and radius, increasing substantially for small thicknesses and radii. Accounting for these small sizes in both radius and thickness is essential for understanding the experimentally observed hole $g$-factor. The contributions to the hole $g$-factor from other effects, such as additional localization of the hole due to electron-hole binding to form the exciton and different choices for materials parameters, have little effect on the value of the hole $g$-factor.

To better understand why the $g$-factor for the ground state (heavy) holes has this strong increase at small QD thicknesses and radii, we have calculated the contributions of hole spin (through the atomic Zeeman splitting), atomic orbital angular momentum and orbital motion in the QD to the hole $g$-factor. The contribution from the hole spin and the atomic orbital angular momentum changes very little with QD dot size as shown in Fig. 7. The large change in the hole $g$-factor comes from a quenching of the angular momentum from orbital motion in small dots. The contribution to the $g$-factor from orbital motion in the QD has the opposite sign, competing against the larger contribution from the spin. As a result, quenching the contribution from orbital motion increases the $g$-factor in small QDs.

In summary, by investigating circularly polarized magneto-PL of type-II submonolayer ZnTe/ZnSe QDs, we observed evidence for long spin-flip time (more than $100 \mathrm{~ns}$ ) of holes in submonolayer QDs, which leads to a non-Boltzmann distribution of exciton spin states. Through quantitative analysis of the Zeeman energy splitting and temperature dependence of the DCP, we obtained $g$-factors of the type-II excitons, electrons, and heavy holes. The $g$-factor of holes in QDs is significantly enhanced due to the submonolayer nature of QDs, and it is strongly dependent on the thickness and radius of QDs, as confirmed by atomistic tightbinding calculations.

\section{ACKNOWLEDGMENTS}

The work was supported in part by the National Science Foundation (NSF) under Award No. DMR-1006050 and the U.S. Department of Energy (DOE) Office of Basic Energy Sciences, Division of Materials Sciences and Engineering under Award No. DE-SC003739. Specifically, sample 
preparation was carried out with DOE support (S.D., M.C.T., and I.L.K.), while optical investigations were performed with support from the NSF (H.J. and I.L.K.). A portion of this work was performed at the National High Magnetic Field Laboratory, which is supported by National Science Foundation (NSF) Cooperative Agreement No. DMR-1157490, the State of Florida, and the U.S. Department of Energy (DOE) (J.L. and D.S.). The modeling and simulation were performed at NIST.

${ }^{1}$ J. M. Kikkawa, I. P. Smorchkova, N. Samarth, and D. D. Awschalom, Science 277, 1284 (1997).

${ }^{2}$ J. A. Gupta, D. D. Awschalom, X. Peng, and A. P. Alivisatos, Phys. Rev. B 60, 8394 (1999).

${ }^{3}$ A. Imamōglu, D. D. Awschalom, G. Burkard, D. P. DiVincenzo, D. Loss, M. Sherwin, and A. Small, Phys. Rev. Lett. 83, 4204 (1999).

${ }^{4}$ D. Loss and D. P. DiVincenzo, Phys. Rev. A 57, 120 (1998).

${ }^{5}$ G. Burkard, D. Loss, and D. P. DiVincenzo, Phys. Rev. B 59, 2070 (1999).

${ }^{6}$ B. J. Witek, R. W. Heeres, U. Perinetti, E. P. A. M. Bakkers, L. P. Kouwenhoven, and V. Zwiller, Phys. Rev. B 84, 195305 (2011).

${ }^{7}$ R. Kotlyar, T. L. Reinecke, M. Bayer, and A. Forchel, Phys. Rev. B 63, 085310 (2001).

${ }^{8}$ A. V. Khaetskii and Y. V. Nazarov, Phys. Rev. B 64, 125316 (2001).

${ }^{9}$ V. N. Golovach, A. Khaetskii, and D. Loss, Phys. Rev. Lett. 93, 016601 (2004).

${ }^{10}$ G. A. Khodaparast, R. E. Doezema, S. J. Chung, K. J. Goldammer, and M. B. Santos, Phys. Rev. B 70, 155322 (2004).

${ }^{11}$ B. Nedniyom, R. J. Nicholas, M. T. Emeny, L. Buckle, A. M. Gilbertson, P. D. Buckle, and T. Ashley, Phys. Rev. B 80, 125328 (2009).

${ }^{12}$ D. Rugar, R. Budakian, H. J. Mamin, and B. W. Chui, Nature 430, 329 (2004).

${ }^{13}$ J. R. Petta, A. C. Johnson, J. M. Taylor, E. A. Laird, A. Yacoby, M. D. Lukin, C. M. Marcus, M. P. Hanson, and A. C. Gossard, Science 309, 2180 (2005).

${ }^{14}$ F. H. L. Koppens, C. Buizert, K. J. Tielrooij, I. T. Vink, K. C. Nowack, T. Meunier, L. P. Kouwenhoven, and M. K. Vandersypen, Nature 442, 766 (2006).

${ }^{15}$ E. Reyes-Gómez, N. Porras-Montenegro, C. A. Perdomo-Leiva, H. S. Brandi, and L. E. Oliveira, J. Appl. Phys. 104, 023704 (2008).
${ }^{16}$ L. M. Woods, T. L. Reinecke, and R. Kotlyar, Phys. Rev. B 69, 125330 (2004).

${ }^{17}$ C. Lü, J. L. Cheng, and M. W. Wu, Phys. Rev. B 71, 075308 (2005).

${ }^{18}$ D. V. Bulaev and D. Loss, Phys. Rev. Lett. 95, 076805 (2005).

${ }^{19}$ D. Klauser, W. A. Coish, and D. Loss, Phys. Rev. B 73, 205302 (2006).

${ }^{20}$ H. Ji, B. Roy, S. Dhomkar, R. T. Moug, M. C. Tamargo, A. Wang, and I. L. Kuskovsky, J. Electron. Mater. 42, 3297 (2013).

${ }^{21}$ B. Roy, A. D. Shen, M. C. Tamargo, and I. L. Kuskovsky, J. Electron. Mater. 40, 1775 (2011).

${ }^{22}$ Y. Kim, Y. Ma, A. Imambekov, N. G. Kalugin, A. Lombardo, A. C. Ferrari, J. Kono, and D. Smirnov, Phys. Rev. B 85, 121403(R) (2012).

${ }^{23}$ M. Willatzen, M. Cardona, and N. E. Christensen, Phys. Rev. B 51, 17992 (1995).

${ }^{24}$ I. L. Kuskovsky, W. MacDonald, A. O. Govorov, L. Mourokh, X. Wei, M. C. Tamargo, M. Tadic, and F. M. Peeters, Phys. Rev. B 76, 035342 (2007).

${ }^{25}$ H. Ji, S. Dhomkar, B. Roy, V. Shuvayev, V. Deligiannakis, M. C. Tamargo, J. Ludwig, D. Smirnov, A. Wang, and I. L. Kuskovsky, J. Appl. Phys. 116, 164308 (2014).

${ }^{26}$ M. J. Snelling, E. Blackwood, C. J. McDonagh, R. T. Harley, and C. T. B. Foxon, Phys. Rev. B 45, 3922(R) (1992).

${ }^{27}$ M. Oestreich and W. W. Ruhle, Phys. Rev. Lett. 74, 2315 (1995).

${ }^{28}$ H. Ji, S. Dhomkar, R. Wu, V. Shuvayev, V. Deligiannakis, M. C. Tamargo, J. Ludwig, Z. Lu, D. Smirnov, A. Wang, and I. L. Kuskovsky, J. Appl. Phys. 119, 224306 (2016).

${ }^{29}$ W. C. Chou, A. Petrou, J. Warnock, and B. T. Jonker, Phys. Rev. B 46, 4316(R) (1992).

${ }^{30}$ L. K. Castelano, D. F. Cesar, V. Lopez-Richard, G. E. Marques, O. D. D. Couto, , Jr., F. Iikawa, R. Hey, and P. V. Santos, Phys. Rev. B 84, 205332 (2011).

${ }^{31}$ Y. V. Terent'ev, M. S. Mukhin, A. A. Toropov, M. O. Nestoklon, B. Y. Meltser, A. N. Semenov, V. A. Solov'ev, and S. V. Ivanov, Phys. Rev. B 87, 045315 (2013).

${ }^{32}$ S. Sancho, M. Chaouache, M. A. Maaref, F. Bernardot, B. Eble, A. Lemaître, and C. Testelin, Phys. Rev. B 84, 155458 (2011).

${ }^{33}$ W. Maier, G. Schmieder, and C. Klingshirn, Z. Phys. B 50, 193 (1983).

${ }^{34}$ X. Ma, G. W. Bryant, and M. Doty, Phys. Rev. B 93, 245402 (2016).

${ }^{35}$ M. Graf and P. Vogl, Phys. Rev. B 51, 4940 (1995).

${ }^{36}$ P. Vogl, H. D. Hjalmarson, and J. D. Dow, J. Phys. Chem. Solids 44, 365 (1983).

${ }^{37}$ S. Adachi, Properties of Group-IV, II-V and II-VI Semiconductors (John Wiley and Sons, West Sussex, 2005). 